\title{
Interfacial Band-Edge Energetics for Solar Fuels Production
}

\author{
Wilson A. Smith ${ }^{1}$, Ian D. Sharp ${ }^{2,3}$, Nicholas C. Strandwitz ${ }^{4}$, Juan Bisquert ${ }^{5,6}$
}

[1] Materials for Energy Conversion and Storage (MECS), Department of Chemical Engineering, Faculty of Applied Sciences, Delft University of Technology, Julianalaan 136, Delft 2628 BL, The Netherlands

[2] Joint Center for Artificial Photosynthesis, Lawrence Berkeley National Laboratory, Berkeley, CA 94720

[3] Chemical Sciences Division, Lawrence Berkeley National Laboratory, Berkeley, CA 94720

[4] Department of Materials Science and Engineering and Center for Advanced Materials and Nanotechnology, Lehigh University

[5] Institute of Advanced Materials (INAM), Universitat Jaume I, 12071 Castello, Spain[6]

Department of Chemistry, Faculty of Science, King Abdulaziz University, Jeddah, Saudi Arabia

\begin{abstract}
Photoelectrochemical (PEC) water splitting has received growing attention as a potential pathway to replace fossil fuels and produce a clean, renewable, and sustainable source of fuel. To achieve overall water splitting and the associated production of solar fuels, complex devices are needed to efficiently capture light from the sun, separate photogenerated charges, and catalyze reduction and oxidation reactions. To date, the highest performing solar fuels devices rely on multi-component systems, which introduce interfaces that can be associated with further performance loss due to thermodynamic and kinetic considerations. In this review, we identify several of the most important interfaces used in PEC water splitting, summarize methods to characterize them, and highlight approaches to mitigating associated loss mechanisms.
\end{abstract}

\section{Introduction}

Direct photoelectrochemical water splitting offers a sustainable way to produce carbon-neutral solar fuels in a potentially cost-efficient and scalable way. To date, many devices have been developed to achieve overall water splitting using only solar irradiation as the energy input and another article in this issue provides a comprehensive summary of experimental demonstrations of such devices. ${ }^{1}$ Many of these systems are based around semiconductor photoelectrodes, which are used to absorb light, separate photogenerated charge carriers, and, in some cases, perform catalysis. Relative energy band alignment of the semiconductor absorbers with the liquid redox couple, catalysts, and other surface bound materials strongly affects the resulting efficiency of solar fuels generation. Therefore, it is critical to understand the energetics of interfaces between photoelectrodes and electrolytes, as well as between solid state materials in semiconductor/catalyst assemblies, under conditions relevant to photocatalysis. 
This review will address the roles that band alignments and semiconductor-liquid junctions play in photoelectrochemical devices and how these band alignments can be modified to enhance performance of solar fuels systems. In particular, we focus the solar production of hydrogen through water splitting, but the same fundamental principles can be applied to the reduction of $\mathrm{CO}_{2}$, only noting the differences in the associated reduction potentials and product selectivity considerations to other, non-hydrogen, forms of fuel. We note that this review is not intended to provide an exhaustive survey of literature relating to surface energetics of photoelectrochemical systems. Rather, the goal is to briefly introduce many of the key concepts and recent advances that drive new directions in measurement, understanding, and control of the energetics of semiconductor/electrolyte and semiconductor/catalyst/electrolyte junctions. Following an introduction of the basic interfacial energetics in photoelectrochemical systems, a selected overview of both conventional and emerging experimental methods for determining work functions and band alignment is presented. Next, the roles of both intrinsic and extrinsic dipoles on surface and interface energetics, as well as opportunities for engineering dipoles to achieve improved open circuit potentials, are discussed. Finally, the impact of solid/solid junctions, particularly those defined by semiconductor/catalyst interfaces, on photoelectrochemical energy conversion is given.

\section{Background and General Considerations}

The function of the semiconductor/electrolyte junction in a water splitting cell is to convert energy from incoming photons into desired chemical substances in the liquid phase. The primary event of light absorption is the production of an electron-hole pair. Subsequently, efficient electron-hole charge separation is required to ensure that the electrochemical reaction for fuel production occurs with a high yield. A number of architectures and configurations with various levels of integration have been explored for solar water splitting. For the case of pure photoelectrochemical (PEC) water splitting (i.e. in the absence of separate buried junction photovoltaic elements), the cell will typically include either a single photoelectrode electrically connected to a metal electrode, as shown in Figure 1(a), or two coupled photoelectrodes, as shown in Figure 1(b). In both cases, the two components are used to drive the required redox reactions for hydrogen and oxygen evolution in physically different locations. 
(a)

E vs. RHE

(eV)

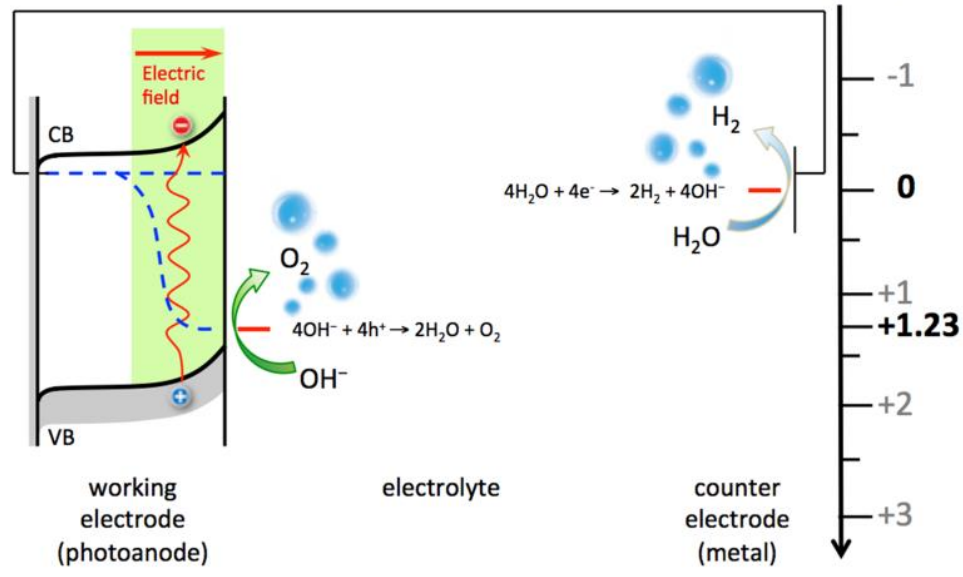

(b)

E vs. RHE

(eV)

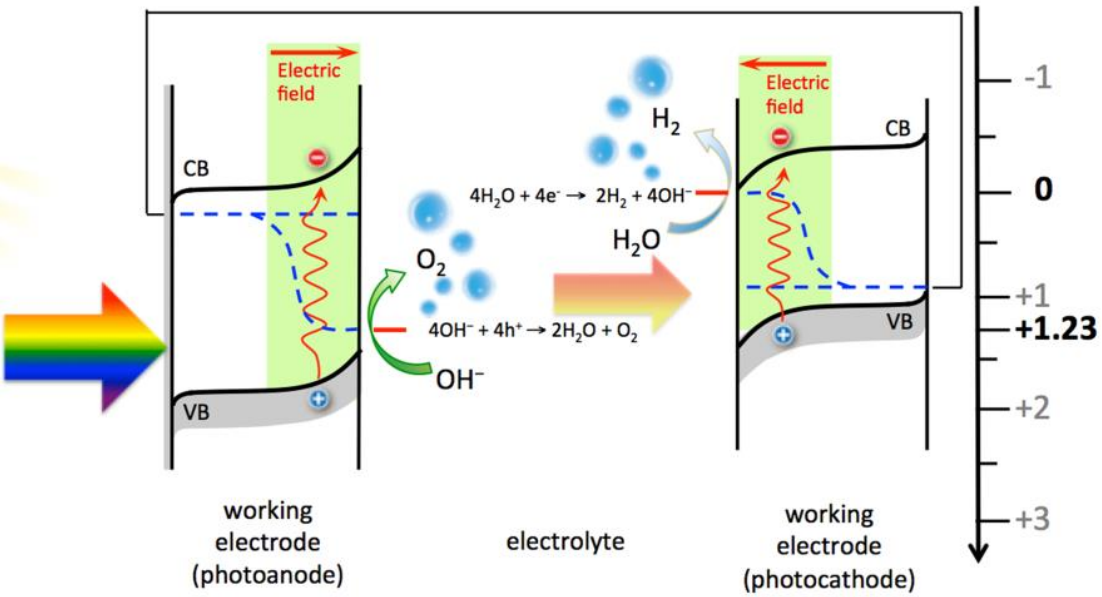

Figure 1: Schematic illustration of (a) a single absorber photoelectrochemical cell with a photoanode and a metal cathode, and (b) a dual absorber system consisting of a photoanode and a photocathode in a tandem configuration. Blue dashed lines represent quasi Fermi levels under illumination. $\mathrm{CB}$ and $\mathrm{VB}$ indicate the conduction and valence bands, respectively. 


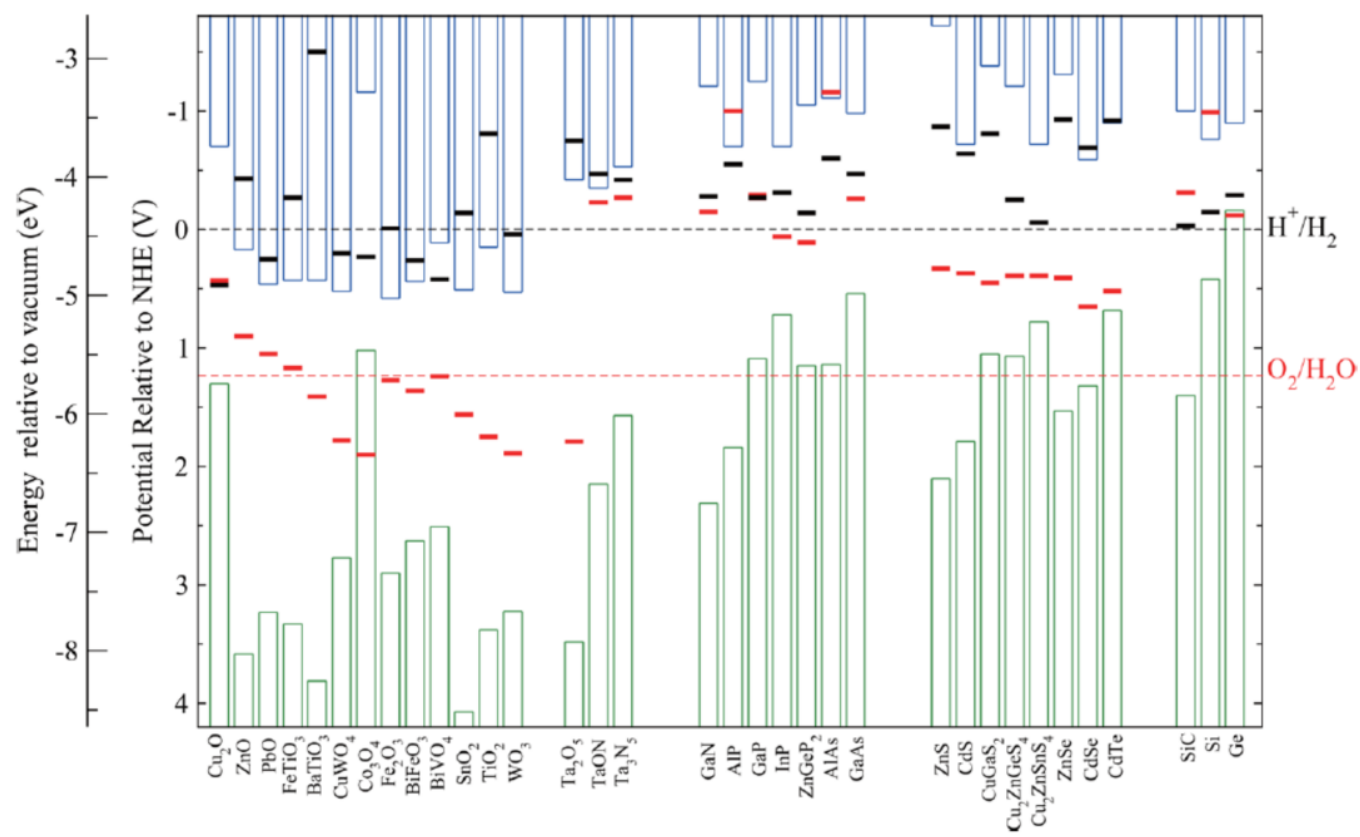

Figure 2: Summary of semiconductor conduction band (blue) and valence band (green) positions at $\mathrm{pH} 0$ relative to the $\mathrm{O}_{2} / \mathrm{H}_{2} \mathrm{O}$ and $\mathrm{H}^{+} / \mathrm{H}_{2}$ redox potentials. For a photoanode to drive the oxygen evolution reaction, its valence band must lie at a more positive potential than the $\mathrm{O}_{2} / \mathrm{H}_{2} \mathrm{O}$ potential and for a photocathode to drive the hydrogen evolution reaction, its conduction band must lie at a more negative potential than the $\mathrm{H}^{+} / \mathrm{H}_{2}$ potential. For a single material to drive overall water oxidation, both conditions must be met. Also shown in the figure are calculated semiconductor oxidation (horizontal red bars) and reduction (horizontal black bars) potentials, which define the thermodynamic stabilities of photoelectrodes against photocorrosion. In this representation, a semiconductor is generally stable against oxidation by photogenerated holes if this oxidation potential lies lower than the valence band position or the $\mathrm{O}_{2} / \mathrm{H}_{2} \mathrm{O}$ potential. Likewise, a material is generally stable against reduction by photogenerated electrons if this reduction potential is higher than the conduction band position or the $\mathrm{H}^{+} / \mathrm{H}_{2}$ potential. Reprinted with permission from Ref. 4. Copyright 2012 American Chemical Society.

photoelectrode, as shown in Figure 1(a). For the case depicted here, the photoelectrode is a photoanode, which is an n-type semiconductor in which photogenerated minority carrier holes flow toward the surface, where they participate in the oxygen evolution reaction by the oxidation of water or hydroxide. Majority carrier electrons are directed to a metallic catalytic electrode, where they reduce protons or water to hydrogen. Charge separation is driven by the electric field in the space charge region under the surface of the electrode, which is established by the energetic alignment of the semiconductor Fermi level and the electrochemical potential of the solution. Therefore, the net steady state effect of light absorption is: (i) the presence of an excess concentration of holes at the surface of the photoanode and with potential given by the valence band maximum, and (ii) an increased concentration of electrons on the surface of the metallic cathode with an energy given by the Fermi level of the metal. These carriers must have sufficient potential to drive the water oxidation and reduction reactions, whose thermodynamic potentials are separated by $1.23 \mathrm{~V}$. For the case of a photoanode-based single junction photoelectrochemical cell, photogenerated minority carriers (holes) will arrive ideally at the surface with a potential given by the energy 
of the valence band at the surface. However, their ability to drive the anodic reaction in a net forward direction is dictated by the quasi-Fermi energy of the holes at the surface; the quasiFermi energy must be more positive in potential than the desired oxidation reaction. ${ }^{2,3}$ On the other hand, electrons will arrive at the metallic cathode with an electrochemical potential corresponding to the majority carrier (electron) Fermi level in the bulk of the photoanode. This electrochemical potential must be at a sufficiently negative potential (i.e., close enough to vacuum level, VL) to drive the reduction of protons. This presents a problem for most metal oxide photoanodes; the Fermi level lies below the conduction band edge, but the conduction band edge is frequently lower in energy than the hydrogen reduction electrochemical potential. This issue is highlighted in Figure 2, which shows a representative summary of conduction band (blue) and valence band (green) positions of a variety of semiconductors at $\mathrm{pH} 0$ relative to the $\mathrm{O}_{2} / \mathrm{H}_{2} \mathrm{O}$ and $\mathrm{H}^{+} / \mathrm{H}_{2}$ redox potentials. ${ }^{4}$

The description above assumes that charge separation occurs with an efficiency of unity, which is never the case due to limitations associated with recombination of electrons and holes within the semiconductor and at its interfaces. However, even if the recombination rate is small, the available carriers at the surface of the electrodes must have sufficient energetic driving force, or overpotential, to perform the water oxidation and reduction reactions at sufficient rates. In practice, this means that even if the requirements for band edge energetic alignment are met, a total photovoltage of $1.7-2 \mathrm{~V}$ must be generated under illumination. ${ }^{5}$ Semiconductors that absorb a large portion of the solar spectrum (with a bandgap of about $2 \mathrm{eV}$ ), will provide an internal photovoltage lower than this value; the quasi-Fermi levels of both electrons and holes cannot approach the band edges at the natural illumination level and the maximum available photovoltage is limited to approximately $\sim \mathrm{E}_{\mathrm{g}} / \mathrm{q}$ $-300 \mathrm{mV}{ }^{6}$ Therefore, a single visible light absorbing semiconductor is not able to deliver sufficient voltage to carry out the oxygen and hydrogen evolution reactions simultaneously. A few wide bandgap semiconductors can support this reaction, but they absorb only a very small fraction of the solar spectrum and are not relevant for practical solar energy conversion applications.

The water splitting cell described above may be coupled with a solid state photovoltaic (PV) element to provide the additional photovoltage required to complete the overall fuel producing reaction. However, the use of semiconductor/electrolyte junctions to generate photovoltage, separate photocarriers, and directly drive photoelectrochemical reactions offers a number of advantages, as well as challenges, compared to solid/solid junctions. Semiconductor/electrolyte junctions can be fabricated without the need for complex, and often expensive, semiconductor deposition and processing steps. Wetting of the electrolyte onto the solid surface can enable facile junction formation over high surface area or polycrystalline materials, and shunt resistances are generally large compared to solid state photovoltaic devices. This last point means that much larger fill factors from structurally imperfect thin films can often be achieved from semiconductor/electrolyte junctions compared to solid/solid junctions using the same material.

Given the advantages of semiconductor-electrolyte junctions, a second approach to ideal photoelectrochemical water splitting, unassisted by external voltage, is to couple a semiconductor photoanode with a semiconductor photocathode, in a tandem cell arrangement, as shown in Figure 1(b). ${ }^{7-11}$ Since a portion of the solar spectrum must be transmitted through 
the top cell, the bandgaps of these materials must be selected to ensure complementary light absorption ranges. For the tandem configuration depicted in Figure 1(b), the photoanode is an n-type semiconductor in which photogenerated minority carrier holes flow toward the surface, where they participate in the oxygen evolution reaction, as described above. The majority carrier electrons are oppositely directed to the back contact, where they recombine with holes from the photocathode. Likewise, the photocathode is a p-type semiconductor in which minority carrier electrons drift to the surface to reduce protons or water to hydrogen and majority carrier holes flow to the back contact where they recombine with electrons from the photoanode.

As for the case of the single photoelectrochemical junction described above, there are strict requirements for the energetics of dual photoelectrochemical junction systems for spontaneous overall water splitting. However, in this case, the energetics of the two different photoelectrodes can be selected for optimal alignment with the respective half reaction potentials (Fig. 2). For a photoanode, the main criterion is that the valence band edge must be deeper (i.e. more positive potential) than the redox potential of the fuel producing reaction, so that the surface collected holes have sufficient oxidation power. For the photocathode, the conduction band minimum must be higher (i.e. more negative potential) than the respective reduction potential.

In addition to the strict thermodynamic requirements for energy level alignment, it is also necessary to generate sufficient overall photovoltage to provide a kinetic driving force for the reactions with high rates. The upper limit to the photovoltage generated is strictly defined by the energetics at the semiconductor/electrolyte interface in absence of other charge transfer asymmetries. In particular, the built-in potential in the semiconductor is ideally defined by the energetic difference between the Fermi energy in the semiconductor and the electrochemical potential in solution. Therefore, it is desired that, for a photoanode, the valence band lies just below (more positive potential than) the $\mathrm{O}_{2} / \mathrm{H}_{2} \mathrm{O}$ potential and, for a photocathode, the conduction band lies just above (more negative potential than) the $\mathrm{H}^{+} / \mathrm{H}_{2}$ potential. In principle, the edge of the conduction band and valence band levels of a semiconductor are given by the fundamental values of electron affinity (EA) and ionization potential (IP), but in practice these are affected by a number of factors. First, EA and IP are intrinsically variable as they depend on the surface termination and its reconstruction, impurities and surface electronic states. Solid materials possess an intrinsic dipole at the surface, where the bulk symmetry is broken. For example for metal oxides, a metal termination leads to a lower and an oxygen termination to a higher electron affinity. ${ }^{12}$ As discussed later in this article, the surface dipole can lead to variations of the band edge position that amount to several hundred meV. ${ }^{13}$ In addition, external surface dipoles can be added that modify the surface energy levels, since in general the electrostatics of the solution are invariant.

The energy level at the surface may also be modulated by adsorbed ionic species, and by the charge of surface states, since these phenomena determine the charging of the Helmholtz layer and hence the Helmholtz potential at the surface. ${ }^{14}$ Surface states near midgap in energy, that are due to the broken bonds or defective stoichiometry at the surface, often determine to a great extent the behavior of the semiconductor interface. ${ }^{15}$ In general, there are two distinct pathways for charge transfer to the solution species: direct transfer from the band edge level, or transfer mediated by trapping at surface states. ${ }^{15}$ Criteria to distinguish 
these mechanisms have been established in terms of the measurements of impedance spectroscopy. ${ }^{16}$ The capture of a minority hole at a surface state of a photoanode and the capture of a minority electron at the surface of a photocathode are loss processes that consequently reduce the available energy for surface reaction. Furthermore, the hole (electron) charged state becomes readily available to capture an electron (hole) from the surface, increasing recombination rates. The existence of surface states has been broadly recognized, and surface treatments are used to decrease their number. ${ }^{17-19}$ Passivation layers may also have the effect of protecting the light-absorbing semiconductor from decomposition.

In practice, obtaining a sizeable photocurrent is a major limitation of many present day water splitting semiconductors, due to inefficiencies in the charge separation steps. This is particularly true for thin film metal oxide and oxynitride materials, in which the crucial point is the collection of minority carrier holes towards the surface. This process is determined by the minority carrier diffusion length, which is in turn dependent on the product of the carrier diffusion coefficient and the recombination lifetime. Another major parameter of the semiconductor is the light absorption length, which is defined as the reciprocal of the absorption coefficient. Only the minority carriers generated closer than one diffusion length from the space charge region near the surface may be collected to contribute to the surface reaction. Hence if the light absorption length is longer than sum of the minority carrier diffusion length and the surface depletion width, carrier collection towards the surface is not efficient.

Minority carrier collection is greatly assisted by a space charge region formed at the surface. As described above, for an n-type semiconductor, the band bends upwards towards the surface, and for a p-type semiconductor it bends downwards (shown at the semiconductor/electrolyte interface in Figure 1(b)). In the depletion region, majority carriers are scarce, hence recombination of minority carriers is greatly reduced. Additionally, in the space charge region the transport rate in opposite directions is enhanced in the form of a drift current by the presence of an electrical field. To design the photoelectrode properties, a number of aspects must be carefully balanced. If the doping level is large, as is usually the case in metal oxide materials, the increased conductivity of majority carriers favors the flow to the back contact, decreasing voltage loss for transport, but the depletion region becomes thinner, which decreases the size of the favorable zone for minority charge collection.

The rate of flow of minority carriers to the surface, assisted by surface depletion, is a necessary consideration, but when carriers do arrive at the surface the rate of the electrochemical reaction is constrained by the interfacial kinetics. Especially in a multielectron process such as the oxygen evolution reaction, which requires the transfer of four holes, accumulation of the holes at the surface will significantly impact the photoelectrode operation. Sluggish kinetics introduce the need for an overpotential; for the case of modern oxygen evolution reaction catalysts, an overpotential of at least $300 \mathrm{mV}$ is typically required..$^{20,21}$ Furthermore, an accumulation of minority carriers enhances greatly the surface recombination and decreases the observed photocurrent. Most bare semiconductor surfaces suffer from poor native catalytic activity and integration of catalyst onto the surface, without adversely affecting the interfacial energetic alignment, is necessary. A catalytic element has the role to improve the surface kinetics for the hole (or electron) transfer reaction. Therefore a semiconductor electrode can be supplemented by additional layers that improve 
reaction rates. Enhanced charge transfer kinetics will decrease the overpotential needed for the onset of photocurrent, and promote faster extraction of minority carriers, that will also result in reduced recombination close to the flatband potential. The effect of enhanced kinetics on the current-potential curves occurs both on improved voltage and fill factor.

In addition to these considerations, the chemical and photochemical stability of bare semiconductor surfaces can severely limit the durability for practical solar fuel generators. The stability challenge is being addressed by (1) selecting thermodynamically stable materials (see Figure 2), (2) applying catalysts that kinetically stabilize the surface against photocorrosion by efficiently extracting charge, or (3) integrating interfacial corrosion protection layers that act as physical barriers between chemically incompatible semiconductors and electrolytes. ${ }^{22-28}$ However, a catalytic element, especially if it is thick on the surface, may affect a number of electronic functional properties of the surface. Hence, the actual operation of surface modifications cannot be easily classified into an energetic or kinetic effect, and this is often a matter of controversy. ${ }^{29}$

The above text serves as an outline for the physical and chemical nature of interfacial band edge energetics with relation to semiconductor based solar water splitting devices. The next section briefly summarizes techniques used for examining the interfacial energetics of semiconductors, with special attention paid to systems adaptable to conducting measurements in an electrolytic environment. After this, we discuss the role of dipolar shifts at photoelectrochemical junctions and how they are used to optimize figures of merit, primarily the photovoltage. We also discuss the role of solid state photovoltaic junctions in solar water splitting. Finally, the role that the liquid contact plays in the optoelectronic performance of photoelectrodes is assessed by comparing buried junctions with photoelectrochemical (semiconductor/liquid) junctions.

\section{Methods for Determining Interfacial Energetics}

Both in situ and ex situ methods are available for determining the impact of dipoles on the energetics of semiconductor surfaces. Under ultrahigh vacuum conditions, ultraviolet photoelectron spectroscopy (UPS) is commonly utilized to determine the work function of a material, which is directly impacted by the presence of dipoles and surface layers. ${ }^{30}$ In this method, the secondary electron cutoff is determined and, with appropriate calibration of the work function of the detector and accelerating bias, provides an absolute measurement of the work function of the sample under vacuum conditions. While this is a powerful analysis method, it is important to note that the surface of the material, and thus its work function, is often significantly different under vacuum, atmospheric, and electrolyte conditions. Therefore, while such vacuum-based measurements are valuable for understanding basic surface modifications and their potential roles for affecting energetic alignment when the material is brought into contact with another phase, it is necessary to exercise caution in developing quantitative portraits of interfacial energy alignment from such measurements alone.

In recent years, considerable advancements have been made in the development of instruments for in situ ambient pressure X-ray photoelectron spectroscopy (APXPS) under controlled gas atmosphere. However, determination of the secondary electron cutoff for work function measurement is based on analysis of photoelectrons with small kinetic energies and 
must be carried out in ultrahigh vacuum. To overcome this challenge, Axnanda and coworkers developed a method for performing in situ work function measurements under gas environments by monitoring the core level shift from Ar gas near the sample surface. ${ }^{31}$ In conventional XPS measurements, core level binding energies are determined relative to the Fermi energy of the material. Therefore, the work function of the sample does not influence core level binding energies, though doping and surface space charge regions do lead to important energy shifts. ${ }^{32}$ However, by analyzing the binding energies of core levels from gas phase molecules near the surface, which are referenced to the vacuum level, it is possible to directly establish the work function. While this approach was only recently developed, it provides significant opportunity for analysis of work functions of materials in exotic environments and under conditions relevant for understanding their function.

Very recently, APXPS methods have been extended to enable operando measurements of electrochemical systems by collecting photoelectrons through a thin liquid layer on the surface of a material that is partially submerged in an electrochemical cell. ${ }^{33,34}$ Because this thin liquid layer is physically continuous and electronically coupled with the bulk electrolyte in the electrochemical cell, measurements can be performed as a function of applied electrochemical potential. This technique was used by Lichterman et al. to determine the energetics of the semiconductor/liquid junction of a $\mathrm{TiO}_{2}$-coated $\mathrm{Si}$ electrode by monitoring core level binding energies of peaks related to the bulk electrolyte, the electrolyte/solid interface, and the solid surface as a function of applied electrochemical potential, as shown in Figure 3. ${ }^{35}$ Their results suggest that interface energetics are dominated by the presence of surface defects in particular applied potential regions. In one regime, defect-induced pinning leads to an applied-potential-independent band bending, whereas other potential regimes show a clear dependence of band bending on applied potential. These results imply that the complicated electronic energetics at the semiconductor/liquid interface are not uniform in different applied potential regions. Therefore, defect concentrations, distributions, and occupations have a significant impact on the behavior of semiconductor/electrolyte junctions. These insights are important for understanding the behavior of real materials systems, which possess defects and non-uniformities that can define interface energetics in ways that are difficult to predict based on idealized, bulk material properties. 


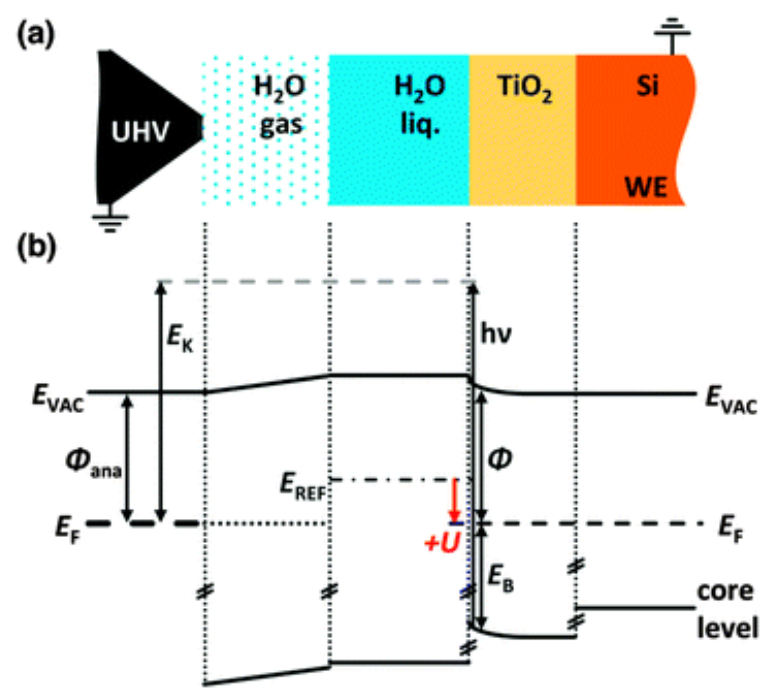

(c)

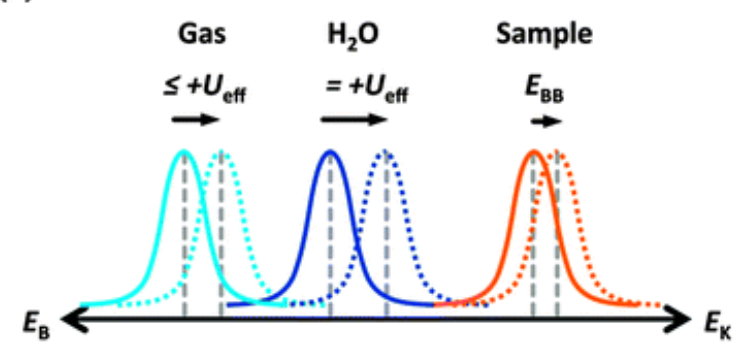

Figure 3: Working set-up of the operando APXPS system used in Ref. 32, with a schematic diagram (top), associated electronic band diagrams (middle), and measured potentials of the $\mathrm{H}_{2} \mathrm{O}$ gas, $\mathrm{H}_{2} \mathrm{O}$ liquid, and working electrode (bottom). $E_{v a c}$ is the vacuum level, $E_{F}$ is the Fermi energy of the sample and analyzer, which are both grounded in the experiment, $E_{K}$ is the kinetic energy of photoelectrons from the sample with respect to the Fermi energy, $\phi_{\text {ana }}$ is the analyzer work function, $\phi$ is the work function of the sample, $h v$ is the incident x-ray photon energy, $U$ is the applied electrochemical potential, $E_{R E F}$ is the reference electrode energy with respect to $E_{v a c}, E_{B}$ is the binding energy under applied electrochemical potential, and $E_{B B}$ is the band bending term. Reproduced from Ref. 35 with permission from The Royal Society of Chemistry.

A second method for the determination of work function is by contact potential difference (CPD) measurement. This measurement typically makes use of a vibrating gold mesh, known as a Kelvin probe, that is capacitively coupled to the surface of the material to be analyzed. The potential drop between a Au reference electrode and the sample is a direct consequence of the difference between sample and reference work functions. This method does not require ultrahigh vacuum conditions, can be implemented in an atomic force microscope for local work function determination (Kelvin probe force microscopy), and can also be used for determination of surface photovoltage by comparing values obtained in dark and under illumination. Therefore, CPD is a useful technique for determining changes of work function and understanding roles of surface dipoles on interfacial energetics, both at the macroscale and the nanoscale. However, it does not provide absolute work function measurements since all values are referenced to the work function of the Au electrode, which can itself vary due to contamination. Although CPD measurements have been performed 
under liquid environments, measurements are complicated by static charge that can lead to erroneous offsets in the extracted work functions and in situ studies have been largely restricted to Kelvin probe-based surface photovoltage measurements. ${ }^{36}$

Another important in situ characterization technique is electrochemical impedance spectroscopy (EIS). Capacitance-voltage measurements of samples in electrolyte are frequently used to construct Mott-Schottky plots $\left(1 / \mathrm{C}^{2}\right.$ vs. E), from which the flat band potential of the semiconductor can be extracted (see below). This method provides direct measurement of the interfacial energetic alignment and can be used to infer the role of interfacial dipoles on the system. However, measurements can be complicated by chemical and electronic inhomogeneity, which are frequently present in thin film materials and lead to non-linear Mott-Schottky plots that preclude determination of interfacial alignment. Further discussion about in situ characterization techniques can be found in an accompanying article in this issue. ${ }^{37}$

In addition to the work function and flat band potential, both the bandgap of the semiconductor and its Fermi level must be known in order to establish a more complete portrait of the interfacial energetics. The most common method for determination of semiconductor bandgaps is by UV-Vis optical absorption spectroscopy. The absorption coefficient is determined via optical transmission or diffuse reflectance measurements as a function of photon energy. The Tauc equation provides the functional dependence of the optical transition strength on photon energy according to:

$$
(\alpha h v)^{n}=A\left(h v-E_{g}\right)
$$

where $\alpha$ is the experimentally measured optical absorption coefficient, $h v$ is the photon energy, $E_{g}$ is the transition energy, and $A$ is a proportionality constant. The exponent, $n$, is equal to $1 / 2$ for indirect transitions and to 2 for direct transitions. Therefore, analysis of plots of $(\alpha h v)^{1 / 2}$ and $(\alpha h v)^{2}$ versus $h v$ can be used to determine the nature of optical transitions (i.e. direct or indirect) and extrapolation of the linear region of the lowest energy transition to the $x$-axis intercept provides a measure of the semiconductor bandgap. ${ }^{38}$.

The bulk Fermi level position relative to the band edges is calculated from the majority carrier concentration. For the case of single crystalline materials or compact thin films with inactive grain boundaries and electrically isolated substrates, free carrier concentrations can be determined via Hall effect measurement. However, many thin film semiconductors used for solar water splitting applications are not well suited for these measurements. An alternative method for determining the dopant concentration is through Mott-Schottky analysis of the ac response of a semiconductor working electrode, measured in a three electrode electrochemical cell in the dark. For an n-type semiconductor, the MottSchottky equation is given by:

$$
\frac{1}{C^{2}}=\frac{2}{\varepsilon \varepsilon_{0} A^{2} e N_{D}}\left(E-E_{f b}-\frac{k_{B} T}{e}\right)
$$

where $C$ is the space charge capacitance, $\varepsilon$ is the dielectric constant, $\varepsilon_{0}$ is the permittivity of free space, $A$ is the area, $e$ is the electronic charge, $N_{D}$ is the effective majority carrier 
concentration, $E$ is the applied electrochemical potential, $E_{f b}$ is the flat band potential, $k_{B}$ is the Boltzmann constant, and $T$ is the temperature. As described above, the $x$-axis intercept of a Mott-Schottky plot provides the flat band potential, and the slope can be used to determine the donor density. The Fermi energy for the n-type semiconductor can then be calculated according to:

$$
E_{C}-E_{F}=k T \ln \left(\frac{N_{C}}{N_{D}}\right)
$$

in which $N_{C}$ is the effective density of states in the conduction band and $\left(E_{C}-E_{F}\right)$ is the energy difference between the conduction band edge and the Fermi energy. In addition, photoemission spectroscopy (either UPS or XPS) can be used to determine the Fermi level within the bandgap. Since the electron binding energy is referenced to the Fermi energy, the binding energy onset of photoemission from the valence band yields the energy difference between the Fermi energy and valence band edge. ${ }^{30}$ However, it is important to note that these measurements yield the surface Fermi level, which is affected by surface band bending and can also be influenced by photovoltages generated under X-ray or ultraviolet illumination during measurement.

\section{Impact of Surface Termination on Interfacial Energetic Alignment (Intrinsic Surface Dipoles)}

Traditionally, the band positions are considered to be defined by the electron affinity of the semiconductor and not experimentally tunable. However, the existence of a dipole layer at the surface can have a considerable impact on the absolute band positions of a semiconductor. Dipoles at the surface of a material can arise from a number of sources, including surface terminal groups, intentionally introduced molecular layers, or the polarization charge of a compound semiconductor. The magnitude of the potential step, $D_{f}$, associated with an interfacial dipole layer is given by:

$$
D_{f}=N m_{z} / \varepsilon \varepsilon_{0}
$$

where $N$ is the number density of dipoles, and $m_{z}$ is the dipole component normal to the plane of the surface. This potential step modifies the work function of the semiconductor, and thus its energetic alignment with the surrounding environment. Therefore, establishing control over stable surface dipole layers has potential to dramatically impact the performance of photoelectrochemical water splitting systems based on semiconductor/electrolyte junctions. Deviations from the ideal photovoltage are typically a consequence of Fermi level pinning and interfacial recombination that necessitate a larger applied bias to ensure that the forward current (i.e. anodic current for photoanodes and cathodic current for photocathodes) dominates over the recombination current. In addition, catalysts must be applied to ensure that the activation energy barrier for the rate limiting step of the reaction is minimized, thereby reducing the overpotential required to achieve a given photocurrent density.

The atomic terminations of semiconductor surfaces can introduce large dipoles that dramatically alter their work functions and, consequently, the expected built-in potential achieved upon contact with electrolyte. This effect has been well studied for a variety of materials, and is exemplified by the stable hydrogen and oxygen terminations that can be 
achieved on the surface of diamond. Though it possesses a wide bandgap of $5.5 \mathrm{eV}$, diamond is a semiconductor with a wide potential window that is useful for fundamental studies of electrocatalysts, as well a platform for electrochemical biosensors. Due to the electronegativity difference between oxygen and carbon, oxygen-terminated diamond possesses a dipole layer with partial negative charge on the oxygen at the surface and partial positive charge on the carbon below. This yields an electron affinity of up to $1.7 \mathrm{eV}$ for oxygen terminated diamond, as shown in Figure $4 .{ }^{39}$ In contrast, hydrogen terminated diamond possesses an oppositely oriented surface dipole due to the electronegativity difference between hydrogen and carbon. The potential step associated with the partial positive charge on the surface hydrogen atoms results in a negative electron affinity of approximately $-1.3 \mathrm{eV}$. This negative electron affinity leads to a number of interesting phenomena. Charge transfer from the diamond to adsorbed water results in formation of a hole accumulation layer and surface conductivity on hydrogen terminated diamond in air. ${ }^{40-42}$ Furthermore, photoelectrons can be ejected from the material via direct photoexcitation from the valence band with sub-bandgap illumination, which enables photochemical reactions on its surface, even in the absence of band-to-band optical transitions. ${ }^{43,44}$ Thus, the surface termination can have a significant impact over the physical properties of the material and the band edge positions of diamond can be changed by approximately $3 \mathrm{eV}$, simply by modification of the surface terminal group. While this is an extreme example, it highlights the importance of understanding - and potentially controlling - surface terminations to promote desired energetic alignment.

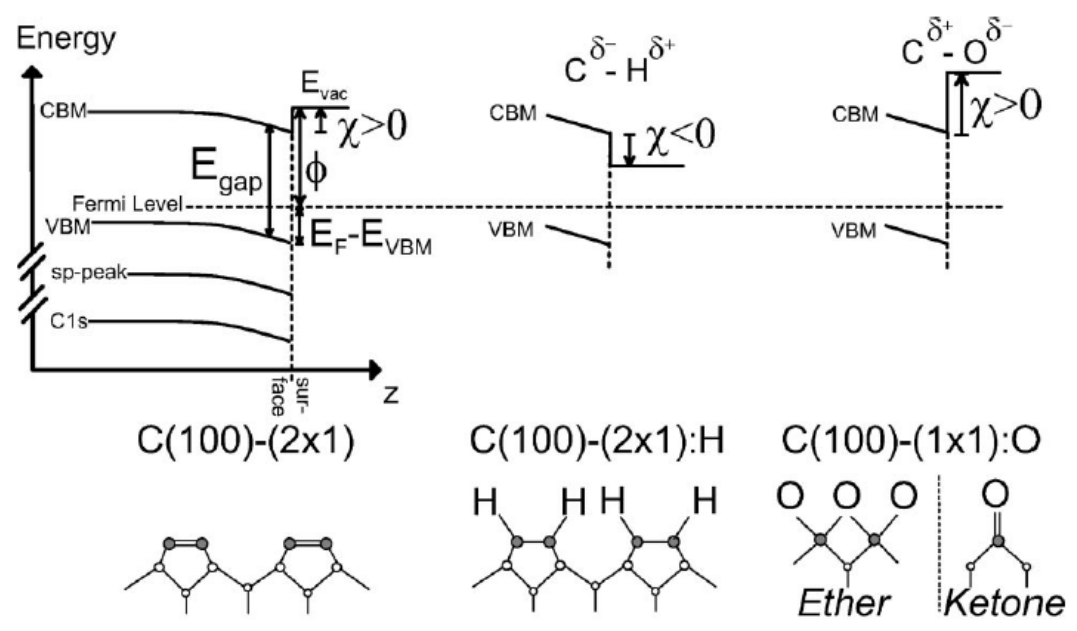

Figure 4: Electronic band diagram for a bare diamond surface (left), a surface that was hydrogenated (middle), and a surface that is oxidized (right) with their associated atomic arrangements (bottom). $E_{v a c}$ is the vacuum energy, $E_{g a p}$ is the diamond bandgap, $E_{F}$ is the Fermi energy, $\phi$ is the work function, CBM is the conduction band minimum, VBM is the valence band maximum at an energy of $E_{V B M}$ relative to vacuum, and $\chi$ is the electron affinity. Reproduced from American Institute of Physics from Ref. 39.

As described above, terminal surface groups can yield dipolar layers that significantly affect the work function of a material. However, once the surface is immersed in electrolyte, the chemical reactivity of these groups must also be considered. In the most common case, 
oxide and other semiconductors naturally possess hydroxyl terminations when contacted with aqueous solution. These groups protonate and deprotonate as a function of $\mathrm{pH}$ according to acid-base equilibrium. ${ }^{45,46}$ Consequently, the potential change of the surface arising from the surface dipole varies with solution $\mathrm{pH}$, in the ideal case with a Nernstian behavior of 59 $\mathrm{mV} / \mathrm{pH}$. Since the surface dipole-induced surface potential and the electrochemical potential change together with $\mathrm{pH}$, the energetics of the semiconductor/electrolyte interface often do not change appreciably with $\mathrm{pH}^{46}$ Therefore, when surface terminal groups are themselves $\mathrm{pH}$ responsive, it is not feasible to obtain significantly more photovoltage from the semiconductor by changing $\mathrm{pH}$. On the other hand, if chemically inert surfaces can be formed, the interfacial energetics will vary with $\mathrm{pH}$. One such surface is hydrogen-terminated diamond, which exhibits a very small $\mathrm{pH}$ sensitivity of $5 \mathrm{mV} / \mathrm{pH}$ due to the chemically inert nature of the terminal $\mathrm{C}-\mathrm{H}$ bonds. ${ }^{47}$ In addition, $2 \mathrm{D}$ compounds, such as transition metal dichalcogenides, have been shown to exhibit a weak flatband potential dependence on $\mathrm{pH}$ due to their layered structure. For these materials, the concentration of edge sites, which are unsaturated and are redox active, plays a crucial role in defining the $\mathrm{pH}$ dependence. ${ }^{48}$ Another example of an inert surface is methylated Si. As demonstrated by Lewis and coworkers, a two-step chlorination and alkylation procedure can be used to saturate all terminal bonds on the Si (111) surface. Much like for the case of hydrogen-terminated diamond, the surface $\mathrm{C}-\mathrm{H}$ bonds exhibit no redox activity and the band positions of $\mathrm{Si}$ are found to be independent of $\mathrm{pH}$, thereby providing opportunity to modify the achievable photovoltage by changing the $\mathrm{pH} .{ }^{49}$ Importantly, the methylation of Si introduces an interfacial dipole, which reduces the work function of the material by $400 \mathrm{meV} .{ }^{50}$ While the dipole is not favorably oriented for improved photoelectrochemical hydrogen evolution, this surface functionalization approach demonstrates the importance of controlling both surface dipole and surface reactivity. As described below, use of more complicated molecular monolayers offers potential for band edge engineering.

\section{Electrostatic Dipole Interfaces (Extrinsic Surface Dipoles)}

While anchoring dipolar molecular species to semiconductor surfaces has been extensively explored and an exhaustive review is outside the scope of this contribution, it is far less commonly utilized in photoelectrochemical systems due to many technical challenges. Therefore, it is useful to identify the specific shortcomings that can arise from using such a system, so that these limitations can be overcome. For example, the dipole effect must be maintained in operational conditions, i.e. it must be chemically stable. This is particularly challenging for the use of molecular monolayers on surfaces for the oxygen evolution reaction (OER) because oxidation of the dipole layers can occur. In addition, penetration of the electrolyte into molecular layers can locally increase the dielectric constant, leading to a substantial reduction in the magnitude of the potential step at the surface. Finally, molecular modification of the surface can also introduce a significant concentration of surface trap states that can increase the surface recombination velocity. If the chemistry of attachment groups or sterics of surface-bound molecules prevent effective interface state passivation, additional approaches, such as use of semiconductors that are inherently robust to defects, incorporation of a window layer for passivation/tunneling, or backfilling with sterically small passivant 
molecules (i.e. methyl groups), ${ }^{51}$ can provide a solution. Furthermore, it is important to note that the effective dipole upon molecular adsorption and not the intrinsic dipole of the isolated molecules must be considered, which calls for the need for complementary theoretical treatment of the system for providing a complete portrait of the interfacial energetics of modified surfaces. ${ }^{52}$

Despite the challenges associated with using dipolar molecular layers to tune photoelectrode energetics, there have been a few successful reports. Kocha and Turner showed that modification of $\mathrm{p}$-GaInP $\mathrm{P}_{2}$ photoelectrodes with 8-quinolinol solution induces a positive shift of the flatband potential by as much as $300 \mathrm{meV}$ in near-neutral electrolyte and an associated shift of the photocurrent onset potential. ${ }^{53}$ The authors found that the role of the molecular species was to induce a change of the Helmholtz layer charge, which shifts the band alignment relative to the unmodified surface. Following this work, Hilal and Turner covalently anchored different pyridyl groups onto GaAs and also observed shifts of the flatband potential of up to $300 \mathrm{meV}$ in darkness. However, while shifts of the photocurrent onset potential were observed, the magnitude of this effect was reduced relative to the values determined from Mott-Schottky analysis in the dark. ${ }^{54}$ Very recently, Olsen, Deutsch, and coworkers investigated the covalent anchoring of phosphonic acid molecules possessing strong dipoles onto $\mathrm{GaInP}_{2}$ photoelectrodes. ${ }^{55}$ Although $\mathrm{GaInP}_{2}$ possesses a bandgap of $1.83 \mathrm{eV}$ that is well suited for efficient water splitting, its band edges are poorly aligned with water redox potentials, as shown in Figure 5. However, by attaching 4-(2,2dicyanovinyl)styrylphosphonic acid (2CVPA) to the surface of $\mathrm{GaInP}_{2}$, the authors were able to favorably shift the band edge positions by as much as $800 \mathrm{meV}$, as measured by photoelectron spectroscopy. Importantly, this yielded a favorable shift of both the flatband potential and the photocurrent onset potential by greater than $200 \mathrm{meV}$ relative to the unmodified electrode. These studies highlight the value of engineering surface dipoles for improving band edge energetics of semiconductor photoelectrodes. However, issues of stability, dielectric screening, and surface recombination must all be addressed in order for the full potential of this approach to be realized. 


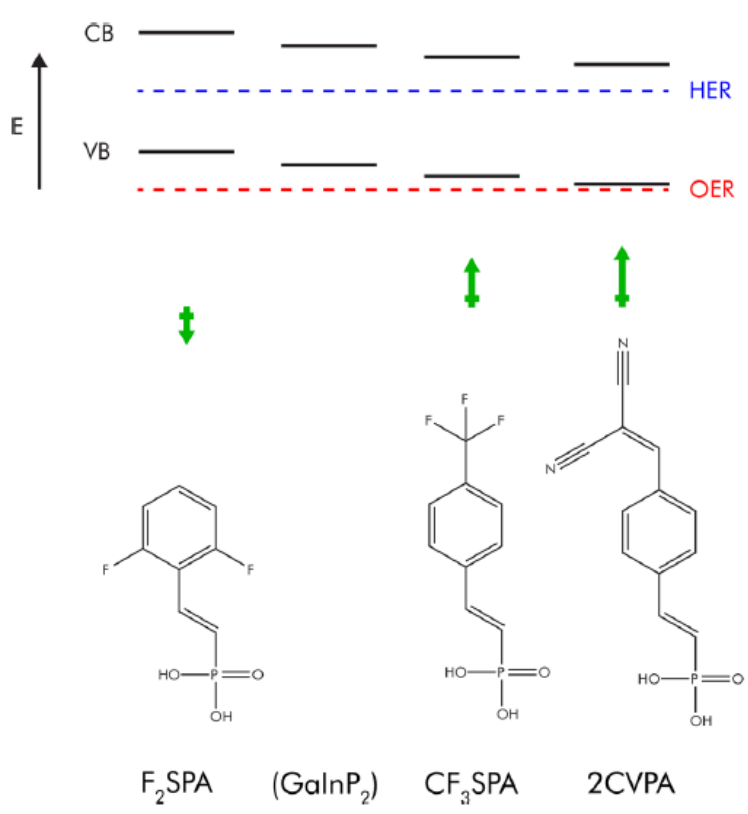

Figure 5: Electronic energies of the conduction band $(\mathrm{CB})$ and valence band $(\mathrm{VB})$ of the bare $\mathrm{GaInP}_{2}$ surface, before and after surface functionalization (top), with respect to the energies for the hydrogen evolution reaction (HER) and oxygen evolution reaction (OER). The band edge energy shifts are given by the extrinsic surface dipoles (with magnitude and direction represented in green) associated with the conjugated phosphonic acids used to modify the surface (bottom). Reprinted with permission from Ref. 55. Copyright 2015 American Chemical Society

In addition, the use of molecular catalysts for water splitting reactions has been widely explored in the past, especially by application of ruthenium polypyridil complexes. The same type of complexes form the basis of dyes for sensitization of $\mathrm{TiO}_{2}$ in order to form dyesensitized solar cells (DSSCs). In this concept, an electron is injected to the semiconductor nanostructure framework while the dye is regenerated by a redox carrier in electrolyte. The same principle has been applied recently in search of a dye-sensitized photoelectrosynthesis cell (DSPEC) capable of water splitting. In this architecture the light absorbing chromophore is integrated with a catalyst for water oxidation. ${ }^{56,57}$ One of the main issues of this approach is to form a stable surface oxide binding of the chromophore-catalyst complex. Very recently, another application of hybrid organic-inorganic solar cells has been developed. It was shown that organic blends used in organic photovoltaics can produce quantitative photocurrent when contacted with an electrolyte. ${ }^{58}$ The formation of protective oxide and catalytic layers is a key step for the development of durable organic PEC devices. ${ }^{59,60}$

\section{Buried junction and adaptive junction interfaces}

For the purpose of this review, we will refer to buried junctions synonymously with photovoltaic (PV) junctions, as recently classified in a report on the taxonomy of solar fuels generators. ${ }^{61}$ Such PV junctions are defined as solid/solid interfaces or homojunctions that determine the degree of band bending (and hence the asymmetry and photovoltage) within a light absorbing semiconductor. Since the built-in electric field is established by the solid/solid interface, the photovoltage generated is electrolyte-invariant. Classic examples of 
such interfaces include metal-semiconductor Schottky contacts, p-n homojunctions, and heterojunctions, which are often identical to those employed in purely light-to-electrical energy conversion PV systems. Properly designed buried junction interfaces result in the formation of a large built-in voltage and open circuit voltage, $V_{o c}$, through solid-solid interfaces rather than solid-liquid interfaces. In contrast, in photoelectrochemical (PEC) junctions, the internal electric fields and photoelectrochemical figures of merit are dependent upon the contacting liquid phase and changes in surface chemistry/dipole formation as described above. In some cases, PV and PEC systems are combined in tandem devices to form PV-biased PEC systems, with a schematic diagram of such a system shown in Figure $6{ }^{62}$ Several of the highest recorded performances for PEC devices (in terms of solar-tohydrogen conversion efficiency, $\eta_{S T H}$ ) have relied, at least in part, on buried junctions. ${ }^{63,64}$ In this discussion, we exclude systems that utilize PV elements connected to separate electrolyzers where the semiconductors are not immersed in the liquid. ${ }^{65,66}$

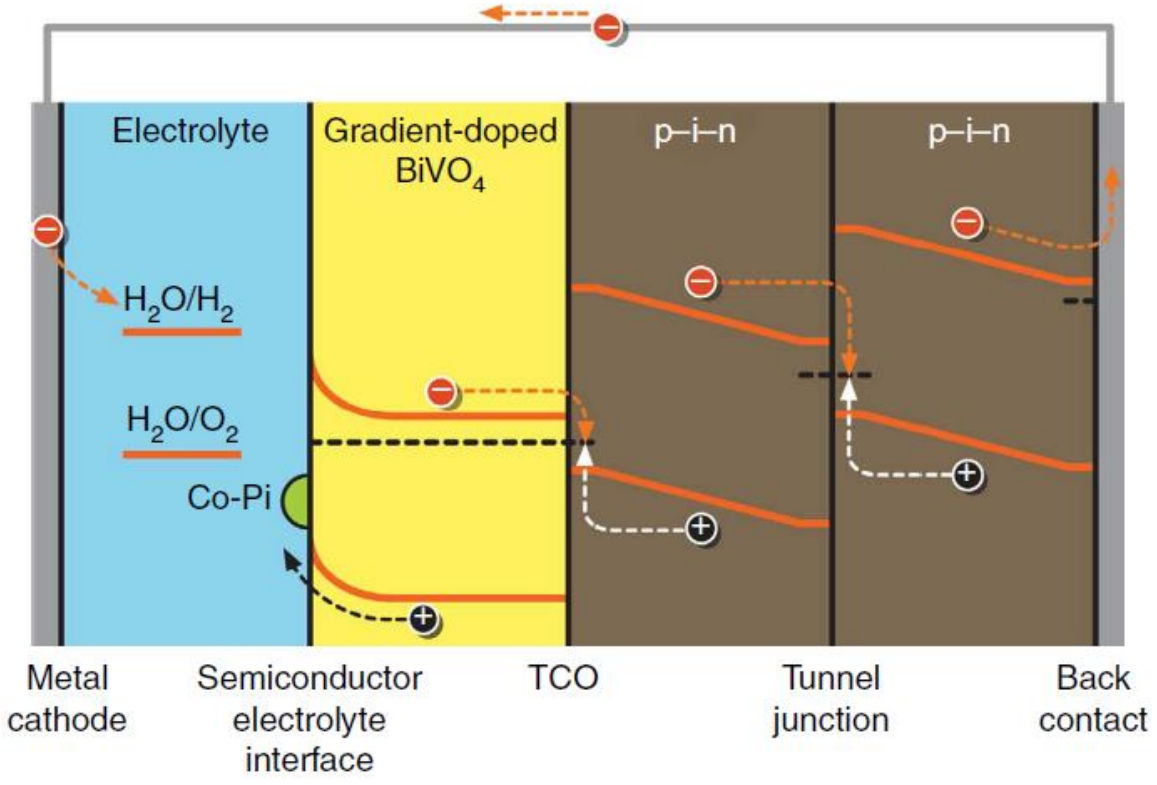

Figure 6: Schematic electronic diagram of a buried junction PV (double junction a-Si) cell powering a $\mathrm{BiVO}_{4}$ photoanode. Adapted with permission from Ref. 62. Copyright 2013 Nature Publishing Group.

PEC junctions are conceptually simpler than PV-based systems and only require contact of a light-absorbing semiconductor with the liquid phase for photoactive junction formation. Therefore, an argument can be made that PEC junctions could be energetically and financially less expensive to produce. However, in water splitting, the redox couple that determines the properties of the PEC junctions is largely fixed (aside from $\mathrm{pH}$ changes or surface dipole engineering), so if a poor junction (e.g., a small barrier height) is formed in a given PEC junction, the solar-to-hydrogen efficiency will be limited. The robustness of PVbased junctions in creating high figures of merit for energy conversion is likely the reason for the recent flurry in publications that use such junctions. ${ }^{67-70}$

Buried PV junctions function as independent voltage generating sources when immersed in solution due to the presence of a highly conductive outer layer. This layer is often a metal, but can also be a highly doped semiconductor or impermeable metal oxide 
layer. $^{69,71}$ In these junctions, the solution equilibrates with the outer conductive layer of the electrode without modifying carrier concentrations (and electric fields) deeper within the semiconductor. Thus, the light absorbing and barrier-forming region of the semiconductor are protected from changes in PV properties by the high density of states near the surface. This allows a fixed photovoltage to be obtained regardless of the electrolyte/redox couple with which the system is in contact.

Since the surfaces of many semiconductors possess poor intrinsic catalytic activity, it is usually necessary to integrate a separate catalyst layer on top of the light absorber. As described above, this catalyst layer can be placed on top of a buried junction, in which case it does not directly affect the established photovoltage, or directly on an unmodified semiconductor surface. However, when continuous, ion-impermeable, and charge conductive catalyst layers are placed on moderately doped semiconductors, buried junctions are generally formed. In some cases, these junctions are undesired since they may interfere with semiconductor/electrolyte PEC junctions that are energetically aligned to provide higher builtin potentials. For cases in which PEC junctions are preferred over PV junctions, but the catalyst interferes with the PEC junction, nanostructured catalyst layers can be employed. As a result of nanostructuring, an inhomogeneous interface can be created, in which semiconductor/catalyst and semiconductor/electrolyte alignments act together to determine the maximum achievable open circuit voltage. When catalyst particles or islands are sufficiently small and well separated from one another, the energetics of the interface are dominated by the semiconductor/electrolyte PEC junction and the system is considered to be in the "pinch-off" regime. ${ }^{72,73}$ In addition to maximizing the photovoltage, nanostructured catalysts have the benefit of reducing parasitic light absorption within the catalyst layer, which can otherwise dramatically reduce the solar energy conversion efficiency. ${ }^{74}$

In some cases, junctions between semiconductors and catalysts have been formed that cannot be clearly categorized as PV or PEC junctions, but may actually be hybrid or "adaptive" junctions, as shown in Figure 7. Conceptually, if the outer catalyst layer has a limited density of states (e.g., a very thin emitter or metal catalyst) or can have its electrochemical potential modified by redox reactions, it can in turn modify the electrostatics of the semiconductor.

a

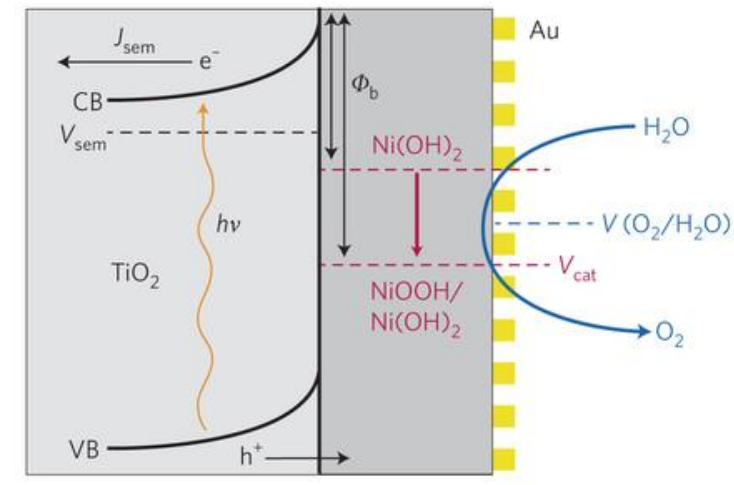

b

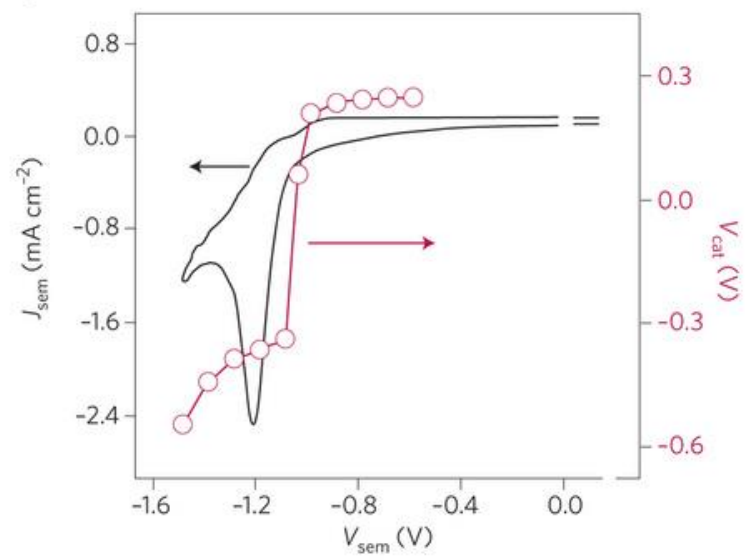

Figure 7: Schematic diagram of an adaptive junction (left) with the associated changes in potential and current density during electrochemical cycling (right). In the figure on the right, as the potential of the semiconductor, $V_{\text {sem }}$, is swept across the given potential range, the left 
axis and black curves represent the current density of the semiconductor, while the red line/points correspond to the measured potential of the catalyst. Adapted with permission from Refs. 75 and 76. Copyright 2014 Nature Publishing Group.

The role of chemical transformation of the catalyst on a photoactive support was explicitly investigated and compared between electrochemically permeable (Ni oxide) and impermeable (Ir oxide) coatings on $\mathrm{TiO}_{2}$ semiconductor absorber. ${ }^{76,77}$ It was found that the oxidation state changes in the $\mathrm{Ni}$ oxide induced a change in barrier height in the $\mathrm{TiO}_{2}$, but the Ir oxide essentially behaved as a metal/semiconductor (buried junction) contact. Since the Ni oxidation state is changed during the course of a cyclic voltammogram, and between the resting and maximum power potentials, the barrier height changes during operation. More recently, this work was expanded to include a broader range of both ion permeable and impermeable electrocatalyst layers on semiconductor surfaces. ${ }^{78}$ The authors found that for ion impermeable catalyst layers, the semiconductor/catalyst junction energetics define the open circuit potential, as expected for a buried junction. In contrast, for ion permeable catalyst layers, the photoelectrochemical properties are defined almost exclusively by the semiconductor/electrolyte junction energetics, independent of the activity of the electrocatalyst layer for water oxidation. ${ }^{76-78}$ This finding could have significant implications in the design of semiconductor/catalyst assemblies exhibiting maximum performance. Other recent examples of what appear to be adaptive junctions include the use of a very thin $(\sim 2 \mathrm{~nm})$ $\mathrm{Ni}$ film on $\mathrm{Si}$ that displayed a larger $V_{o c}$ than thicker ( $>5 \mathrm{~nm}$ ) films. ${ }^{79}$ Also, the functionalization of $\mathrm{Si}$ with manganese oxide $(10 \mathrm{~nm})$ showed variability in $V_{o c}$ with variations in solution potential, indicating this was not simply a buried junction. ${ }^{80}$ Presumably, in these adaptive junctions, the ultimate barrier height that can be achieved is dictated by characteristics of the surface layer (catalyst) when it reaches a steady-state condition with the liquid redox couple at the maximum power point.

\section{Conclusions}

Preserving a large photovoltage in light absorbing semiconductors is critical to achieving high performance PEC water splitting. In this review we have discussed the behavior of interfacial band edge energies and how they are modified by various surface modifications and electrochemical processes. Such modifications may allow for optimization of solar-to-hydrogen conversion efficiencies. These methods should be compared in detail to understand the fundamental aspects, which overlap, and how they can possibly be combined in an optimal way. Furthermore, new approaches should be discussed and evaluated that take into account the strict materials and opto-electronic properties needed for a successful solid/solid or solid/electrolyte interface that allows for high kinetic charge transfer at low overpotentials.

\section{Acknowledgements}

The authors thank Dr. Eric Miller for the inspiration to compile this review, and the members of the DOE Photoelectrochemical Working Group for helpful comments, suggestions, and 
discussions. WAS greatly acknowledges funding support from the FOM/NWO/Shell Program on $\mathrm{CO}_{2}$-neutral Fuels (Project - APPEL). IDS was supported by the Joint Center for Artificial Photosynthesis, a DOE Energy Innovation Hub, supported through the Office of Science of the U.S. Department of Energy under Award Number DE-SC0004993. NCS acknowledges start-up funds from Lehigh University. JB thanks financial support from GeneralitatValenciana (ISIC/ 2012/008). A summary version of this review paper (DOI: $\boldsymbol{T B D}$ ) can be found on the working group website (http://energy.gov/eere/fuelcells/photoelectrochemical-working-group).

\section{References}

(1) J. W. Ager III, M. Shaner, K. Walczak, I.D. Sharp and S. Ardo, Energy Environ. Sci., 2015, DOI: 10.1039/C5EE00457H

(2) A. Kumar, P.G. Santangelo and N.S. Lewis, J. Phys. Chem, 1992, 96, 834-842.

(3) P. Salvador, J. Phys. Chem. B, 2001, 105, 6128-6141.

(4) S. Chen and L.-W. Wang, Chem. Mat., 2012, 24, 3659.

(5) M.G. Walter, E.L. Warren, J.R. McKone, S.W. Boettcher, Q. Mi, E.A. Santori and N.S. Lewis, Chem. Rev., 2010, 110, 6446-6473.

(6) S.M. Sze, Physics of Semiconductor Devices, 1969, New York: Wiley.

(7) M.F. Weber and M.J. Dignam, J. Electrochem. Soc., 1984, 131, 1258-1265

(8) J. R. Bolton, S. J. Strickler and J. S. Connolly, Nature, 1985, 316, 495-500.

(9) J. Brillet, J. H. Yum, M. Cornuz, T. Hisatomi, R. Solarska, J. Augustynski, M. Graetzel and K. Sivula, Nat. Photonics, 2012, 6, 824-828.

(10) S. Hu, C. Xiang, S. Haussener, A. D. Berger and N. S. Lewis, Energy Environ. Sci., 2013, 6, 2984-2993.

(11) H. Doscher, J. F. Geisz, T. G. Deutsch and J. A. Turner, Energy Environ. Sci., 2014, 7, 2951-2956.

(12) W. Jaegermann, Mod. Asp. Electrochem., 1996, 30, 1-186.

(13) A. Hankin, J.C. Alexander and G.H. Kelsall, Phys. Chem. Chem. Phys. 2014, 16, 16176-16186.

(14) S.R. Morrison, Electrochemistry at Semiconductor and Oxidized Metal Electrodes; Plenum Press: New York, 1980.

(15) B. Klahr, S. Gimenez, F. Fabregat-Santiago, T.W. Hamann, J. Bisquert, J. Am. Chem. Soc., 2012, 134, 4294-4302.

(16) L. Bertoluzzi, P. Lopez-Varo, J.A.J. Tejada and J. Bisquert, J. Mater. Chem.

\section{A., 2015, Advance article (DOI:10.1039/C5TA03210E)}

(17) C. Du, M. Zhang, J.-W. Jang, Y. Liu, G.-Y. Liu and D. Wang, J. Phys. Chem. C., 2014, 118, 17054-17059.

(18) O. Zandi and T.W. Hamann, J. Phys. Chem. Lett., 2014, 5, 1522-1526.

(19) J. Zhao and F.E. Osterloh, J. Phys. Chem. Lett., 2014, 5, 782-786.

(20) C.C.L. McCrory, S. Jung, J. C. Peters and T.F. Jaramillo, J. Amer. Chem. Soc., 2013, 135, 16977.

(21) C.C.L. McCrory, S. Jung, I. M. Ferrer, S. M. Chatman, J. C. Peters and T.F. Jaramillo, J. Amer. Chem. Soc., 2015, 137, 4347. 
M.J. Kenney, M. Gong, Y. Li, J.Z. Wu, J. Feng, M. Lanza and H. Dai, Science, 2013, 342, 836-840.

(23) M. H. Lee, K. Takei, J. Zhang, R. Kapadia, M. Zheng, Y.-Z. Chen, J. Nah, T.S. Matthews, Y.-L. Chueh, J.W. Ager, and A. Javey, Angew. Chem., 2012, 124, 1091810922.

(24) Y. W. Chen, J. D. Prange, S. Duhnen, Y. Park, M. Gunji, C.E.D. Chidsey and P.C. McIntyre, Nat. Mat., 2011, 10, 539-544.

(25) Y. Lin, C. Battaglia, M. Boccard, M. Hettick, Z. Yu, C. Ballif, J.W. Ager and A. Javey, Nano Lett., 2013, 13, 5615-5618.

(26) A. Paracchino V. Laporte, K. Sivula, M. Gratzel and E. Thimsen, Nat. Mat., 2011, 10, 456-461.

(27) B. Seger, T. Pedersen, A.B. Laursen, P.C.K. Vesborg, O. Hansen and I.

Chorkendorff, J. Am. Chem. Soc., 2013, 135, 1057.

(28) S. Hu, N.S. Lewis, J. W. Ager, J. Yange, J. R. McKone, and N. C. Strandwitz, J. Phys. Chem. C, 2015 under review.

(29) D.R. Gamelin, Nat. Chem., 2012, 4, 965-967.

(30) D. Cahen and A. Kahn, Adv. Mat., 2003, 15, 271-277.

(31) S. Axnanda, M. Scheele, E. Crumln, B. Mao, R. Chang, S. Rani, M. Faiz, S.

Wang, A. P. Alivisatos and Zhi Liu, Nano Lett., 2013, 13, 6176-6182.

(32) D. Cahen and A. Kahn, Adv. Mat., 2003, 15, 271-277.

(33) O. Karslioglu, S. Nemsak, I. Zegkinoglou, A. Shavorskly, M. Hartl, F.

Salmassi, E.M. Gullikson, M.L. Ng, Ch. Rameshan, B. Rude, D. Bianculli, A.A.

Cordones, S. Axnanda, E.J. Crumlin, P.N. Ross, C.M. Schneider, Z. Hussain, Z. Liu,

C.S. Fadley and H. Bluhm, Faraday Discuss., 2015, advance article.

(34) S. Axnanda, E..J. Crumlin, B. Mao, S. Rani, R. Chang, P.G. Karisson, M.O.M.

Edwards, M. Lundqvist, R. Moberg, P. Ross, Z. Hussain and Z. Liu, Sci. Rep., 2015, 5, 9788 .

(35) M. F. Lichterman, S. Hu, M.H. Richter, E.J. Crumlin, S. Axnanda, M. Favaro, W. Drisdell, Z. Hussain, T. Mayer, B. S. Brunschwig, N. S. Lewis, Z. Liu and H.-J. Lewrenz, , Energy Environ. Sci. 2015, accepted article.

(36) S. Bastide, D. Gal, D. Cahen and L. Kronik, Rev. Sci. Instrum., 1999, 70, 4032.

(37) D. Esposito et al., Energy Environmental. Sci., 2015, accepted article.

(38) P.Y. Yu and M. Cardona, Fundamentals of Semiconductors ( $2^{\text {nd }}$ Ed.), 1999, Springer, Berlin.

(39) F. Maier, J. Ristein and L. Ley, Phys. Rev. B, 2001, 64, 165411.

(40) V. Chakrapani, J.C. Angus, A.B. Anderson, S.D. Wolter, B.R. Stone rand G.U. Sumanasekera, Science, 2007, 318, 1424-1430.

(41) F. Maier, M. Riedel, B. Mantel, J. Ristein and L.Ley, Phys. Rev. B, 2000, 85, 3472.

(42) J.A. Garrido, A. Hartl, M. Dankerl, A. Reitinger, M. Eickhoff, A. Helwig, G.

Muller and M. Stutzmann, J. Am. Chem. Soc., 2008, 130, 4177-4181.

(43) T. Strother, T. Knickerbocker, J.N. Russell, Jr., J.E. Butler, L. M. Smith and R.J. Hamers, Langmuir, 2002, 18, 968-971. 
(44) B. M. Nichols, J.E. Butler, J.N. Russell, Jr. and R.J. Hamers, J. Phys. Chem. B, 2005, 109, 20938-20947.

(45) H. Gerischer, Electro. Acta, 1989, 34, 1005-1009.

(46) M.G. Walter, E.L. Warren, J.R. McKone, S.W. Boettcher, Q. Mi, E.A. Santori and N.S. Lewis, Chem. Rev., 2010, 110, 6446-6473.

(47) M. Danekerl, A. Reitinger, M. Stutzman and J.A. Garrido, Phys. Status Solidi $R R L, 2008,2,31-33$.

(48) H.J. Lewerenz, H. Gerischer and M. Lubke, J. Electrochem. Soc., 1984, 131, 100-104.

(49) T. W. Hamann and N.S. Lewis, J. Phys. Chem. B, 2006, 110, 22291-22294.

(50) R. Hunger, R. Fritsche, B. Jaeckel, W. Jaegermann, L.J. Webb and N.S.

Lewis, Phys. Rev. B, 2005, 72, 045317.

(51) K.T. Wong and N.S. Lewis, Acc. Chem. Res., 2014, 47, 3037-3044.

(52) J. B. Rivest, G. Li, I.D. Sharp, J.B. Neaton and D. J. Milliron, J. Phys. Chem., Lett., 2014, 5, 2450-2454.

(53) S.S. Kocha and J.A. Turner, J. Electrochem. Soc., 1995, 142, 2625-2630.

(54) H. S. Hilal and J.A. Turner, Electrochem. Acta, 2006, 51, 6487-6497.

(55) B.A. MacLeod, K.X. Steirer, J.L. Young, U. Koldemir, A. Sellinger, J.A.

Turner, T.G. Deutsch and D.C. Olson, ACS Appl. Mater. Interfaces, 2015, 7, 1134611350.

(56) J.J. Concepcion, R.L. House, J.M. Papanikolas and T.J. Meyer, Proc. Nat. Acad. Sci. USA, 2012, 109, 15560-15564.

(57) Y. Zhao, J.R. Swierk, J.D. Megiatto, B. Sherman, W.J. Youngblood, D. Qin, D.M. Lentz, A.L. Moore, T.A. Moore, D. Gust and T.E. Mallouk, Proc. Nat. Acad. Sci. USA, 2012, 109, 15612-15616.

(58) A. Guerrero, M. Haro, S. Bellani, M.R. Antognazza, L. Meda, S. Gimenez and J. Bisquert, Energy Environ. Sci., 2014, 7, 3666-3673.

(59) T. Bourgeteau, D. Tondelier, B. Geoggroy, R. Brisse, R. Cornut, V. Artero and B. Jousselme, ACS Appl. Mat. Inter., 2015, 7, 16395-16403.

(60) M. Haro, C. Solis, G. Molina, L. Otero, J. Bisquert, S. Gimenez and A.

Guerrero, J. Phys. Chem. C., 2015, 119, 6488-6494.

(61) A. C. Nielander, M. R. Shaner, K. M. Papadantonakis, S. A. Francis and N. S. Lewis, Energy Environ. Sci., 2015, 8, 16-25.

(62) F.F. Abdi, L. Han, A.H.M. Smets, M. Zeman, B. Dam and R. van der Krol, Nat. Comm., 2013, 4, 2195.

(63) O. Khaselev and J. A. Turner, Science, 1998, 280, 425-427.

(64) R.E. Rocheleau, E.L. Miller and A. Misra, Energy Fuels, 1998, 12, 3-10.

(65) S. Licht, B. Wang, S. Mukerji, T. Soga, M. Umeno and H. Tributsch, J. Phys. Chem. B, 2000, 104, 8920.

(66) J. Luo, J.-H. Im, M.T. Mayer, M. Schreier, M.K. Nazeeruddin, N.-G. Park, S.

David Tilley, H.J. Fan and M. Gratzel, Science, 2014, 345, 1593-1596.

(67) S. Hu, M. R. Shaner, J. A. Beardslee, M. Lichterman, B. S. Brunschwig, and N. S. Lewis, Science, 2014, 344, 1005. 
(68) E.L. Warren, S.W. Boettcher, M.G. Walter, H.A. Atwater and N.S. Lewis, J. Phys. Chem. C, 2011, 115, 594-598.

(69) S.W. Boettcher, E.L. Warren, M.C. Putnam, E.A. Santori, D. Turner-Evans, M.D. Kelzenberg, M.G. Walter, J.R. McKone, B.S. Brunschwig, H.A. Atwater and N.S. Lewis, J. Am. Chem. Soc., 2011, 133, 1216-1219.

(70) I.A. Digdaya, L. Han, T.W.F. Buijs, M. Zeman, B. Dam, A.H.M. Smets and W.A. Smith, Energy Environ. Sci., 2015, 8, 1585-1593.

(71) G. Hodes, L. Thompson, J. DuBow and K. Rajeshwar, J. Am. Chem. Soc., 1983, 105, 324-330.

(72) R.C. Rossi and N.S. Lewis, J. Phys. Chem. B, 2001, 105, 12303.

(73) M. Szklarczyk and J.O.M. Bockris, J. Phys. Chem., 1984, 88, 1808.

(74) L. Trotochaud, T.J. Mills and S.W. Boettcher, J. Phys. Chem. Lett., 2013, 4, 931-935.

(75) T.W. Hamann, Nat. Mat. 2014, 13, 3-4.

(76) F. Lin and S.W. Boettcher, Nat. Mat., 2014, 13, 81-86.

(77) T.J. Mills, F. Lin and S.W. Boettcher, Phys. Rev. B, 2014, 112, 148304.

(78) F. Lin, B.J. Bachman and S.W. Boettcher, J. Phys. Chem. Lett., 2015, 6, 24272433.

(79) M.J. Kenney, M. Gong, Y. Li, J.Z. Wu, J. Feng, M. Lanza and H. Dai, Science, 2013, 342, 836-840.

(80) N.C. Strandwitz, D.J. Comstock, R.L. Grimm, A.C. Nichols-Nielander, J. Elam and N.S. Lewis, J. Phys. Chem. C, 2013, 117, 4931-4936. 